\title{
Qualidade fisiológica de sementes de palmiteiro-vermelho em função da desidratação e do armazenamento
}

\author{
Cibele C Martins' ${ }^{1}$; Marilene LA Bovi ${ }^{2}$; João Nakagawa ${ }^{1}$ \\ ${ }^{1}$ UNESP, FCA, Dep ${ }^{\text {to }}$ Produção Vegetal, C Postal 237, 18603-970 Botucatu-SP; ${ }^{2}$ IAC, Centro de Horticultura, Unidade Plantas Tropicais, \\ C.Postal 28, 13012-970 Campinas-SP; cibele@fca.unesp.br
}

\section{RESUMO}

Para algumas espécies com sementes recalcitrantes, como o palmito-vermelho (Euterpe espiritosantensis Fernandes), a secagem parcial favorece a manutenção da viabilidade durante o armazenamento. O objetivo deste trabalho foi verificar o efeito da desidratação e do armazenamento na germinação e vigor das sementes de palmito-vermelho. Dois lotes de sementes (L1 e L2) foram colocados para secar por 0; 20 e 40 horas (três sublotes) em câmara seca e armazenados a $15^{\circ} \mathrm{C}$ em sacos plásticos fechados. Foi realizado um ensaio em delineamento inteiramente casualizado para cada lote de semente, com os tratamentos dispostos em fatorial 3 × 6 (L1) e $3 \times 10$ (L2), correspondendo a três tempos de secagem e seis ou dez semanas de armazenamento. A qualidade das sementes foi avaliada a cada seis semanas, durante 30 e 54 semanas para respectivamente L1 e L2, por meio do teor de água (U), porcentagem de germinação $(G)$ e vigor (primeira contagem e índice de velocidade de germinação). O teor de água das sementes de ambos os lotes foi em média de aproximadamente de 46; 40 e 36\%, respectivamente, para $0 ; 20$ e 40 horas de secagem, sem alterações importantes em função do período de armazenamento. Para sementes não-armazenadas, a secagem reduziu de modo significativo o vigor, mas não comprometeu a germinação. O lote L1 apresentou sementes viáveis em todos os sublotes até o final do armazenamento $(\mathrm{G}=71 ; 61 \mathrm{e}$ $45 \%$ para $0 ; 20$ e 40 horas, respectivamente). As sementes do lote L2 apresentaram perda total da viabilidade por volta da $30^{\mathrm{a}}$.semana apenas no sublote onde foram secas por 40 horas $(U=36 \%)$. Nos demais sublotes, as sementes se mantiveram viáveis até 54 semanas de armazenamento $(\mathrm{G}=46 \%)$. A secagem parcial reduziu o vigor e diminuiu a longevidade das sementes. Com base nos resultados obtidos pode-se concluir que sementes de E. espiritosantensis devem ser armazenadas sem secagem, com 45-46\% de teor de água.

Palavras-chave: Euterpe espiritosantensis Fernandes, sementes recalcitrantes, germinação, vigor, palmeira.

\section{ABSTRACT}

Physiological quality of Euterpe espiritosantensis seeds as affected by partial drying and storage

Euterpe espiritosantensis seeds are recalcitrant, presenting sensibility to dehydration and difficulty for storage. In some species with recalcitrant seeds, partial drying favors the maintenance of viability during storage. The objective of this research was to study the effects of partial drying and storage on germination and vigor of E. espiritosantensis seeds. Two seed lots (L1 and L2) were dried for $0 ; 20$, and 40 hours in a dry chamber and stored at $15^{\circ} \mathrm{C}$ in closed plastic bags. An assay for each seed lot was carried out on a completely randomized design, with treatments arranged in an $3 \times 6$ (L1) and $3 \times 10$ (L2) factorial scheme, corresponding to three seed drying periods and six or ten weeks of storage. Seed quality was evaluated every six weeks, during 30 and 54 weeks for L1 and L2, respectively. Seed water content (U), percentage of germination (G) and vigor (first counting of germination test and index germination rate) were assessed. Water content during storage, were, in both lots, on average $46 ; 40$, and $36 \%$, respectively, for $0 ; 20$, and 40 hours of drying, without important changes due to storage period. For nonstored seeds, drying reduced significantly vigor, but did not interfere with germination. Seed lot L1 presented viable seeds in all sub lots until the end of the storage period $(\mathrm{G}=71,61$ and $45 \%$ for $0 ; 20$ and 40 hours, respectively). Seeds on lot L2 lost viability completely in the $30^{\text {th }}$ week in the 40 hours of drying treatment $(\mathrm{U}=36 \%)$. Seeds in other treatments remained viable up to 54 weeks of storage $(\mathrm{G}=$ $46 \%$ ). Partial drying reduced vigor and decreased seed longevity. Seeds from E. espiritosantensis can be stored without drying, with $45-46 \%$ of water content.

Keywords: Euterpe espiritosantensis Fernandes, recalcitrant seeds, germination, vigor, palm tree.

\section{(Recebido para publicação em 3 de janeiro de 2006; aceito em 20 de abril de 2007)}

$\mathrm{O}$ palmito-vermelho (Euterpe espiritosantensis Fernandes) é espécie nativa da Floresta Tropical Atlântica, cuja propagação dá-se exclusivamente por sementes. Formações florestais naturais dessa planta foram encontradas na região de Santa Teresa, no estado do Espírito Santo (Fernandes, 1989) e em Ituberá e Itabuna, no Estado da Bahia (Bovi et al., 1987, 1994). A crescente devastação do seu habitat, associada à derrubada de plantas para a extração de palmito, tornam de interes- se a produção de sementes e mudas dessa espécie para a recomposição da flora nativa e, ainda, para pesquisas visando à exploração comercial da espécie para produção de palmito (olerícola não-convencional) ou como planta ornamental.

Sabe-se que as sementes do palmito-vermelho apresentam grande dificuldade em sua conservação, tendo sido classificadas como recalcitrantes (Martins et al., 1999a, 2000) devido à sensibilidade à desidratação, às temperaturas subzero de armazenamento e à curta longevidade. Deste modo, para serem armazenadas, as sementes do gênero Euterpe necessitam ser acondicionadas em embalagens impermeáveis, usualmente sacos plásticos, para evitar a desidratação progressiva e a conseqüente perda de viabilidade, e mantidas a temperaturas entre 5 e $20^{\circ} \mathrm{C}$ (Bovi et al., 1987; Andrade, 1994; Nodari et al., 1998; Martins et al., 2000; Nascimento, 2006). Porém, mesmo utilizando essas condições favoráveis de armazenamento, observou-se que as se- 
mentes do palmito-vermelho apresentaram redução da viabilidade após um mês (Martins et al., 2000).

Segundo Barbedo \& Marcos Filho (1998), a conservação de sementes recalcitrantes poderia ser favorecida por métodos que propiciassem a paralização ou a limitação, ao máximo possível, do crescimento do eixo embrionário, mantendo-se a semente com fornecimento de água suficiente para evitar sua desidratação abaixo do teor de água crítico. Resultados de pesquisas com sementes de cacau e seringueira, também recalcitrantes, indicam que a utilização de uma técnica de secagem parcial das sementes favorece a manutenção da qualidade das mesmas durante o armazenamento (Hor et al., 1984; Chin, 1994; Bilia, 1997; Gentil, 2003; Nascimento, 2006).

Diante do exposto, este trabalho visou verificar o efeito da secagem inicial parcial e do tempo de armazenamento sobre a germinação e o vigor das sementes de palmito-vermelho.

\section{MATERIAL E MÉTODOS}

Frutos maduros de palmiteiro-vermelho foram coletados manualmente, na EE do Instituto Agronômico de Campinas (IAC), localizada no município de Ubatuba (SP) $\left(23^{\circ} 06^{\prime} \mathrm{S}, 4^{\circ} 03^{\prime} \mathrm{W}, 6 \mathrm{~m}\right.$ acima do nível do mar), por dois anos seguidos, formando dois lotes distintos. O primeiro lote (L1) foi composto por sementes colhidas de 19 plantas matrizes, em outubro de 1998 e, o segundo lote (L2), por sementes coletadas de 17 plantas matrizes em agosto de 1999. Imediatamente após a colheita, cada lote foi transportado em embalagem de polietileno até o Laboratório de Análise de Sementes da UNESP, Campus de Botucatu (SP), onde os frutos foram despolpados por meio de fricção em peneira de malha de aço, sob água corrente.

As sementes mal formadas, danificadas ou abaixo do tamanho médio foram eliminadas. As sementes foram colocadas para secar por 0; 20 e 40 horas (três sublotes) em câmara seca (temperatura de $22^{\circ} \mathrm{C}$ e umidade relativa de $38 \%$ ), visando à obtenção de teores distintos de água nos três sublotes.

Tabela 1. Teor de água em porcentagem de dois lotes de sementes de palmito-vermelho em função de períodos de secagem e armazenamento (Water content, in percentage, of two seed lots of Euterpe espiritosantensis as affected by drying period and storage). Botucatu, UNESP, 1998/2000.

\begin{tabular}{|c|c|c|c|c|c|c|}
\hline \multirow{3}{*}{$\begin{array}{l}\text { Armazenamento } \\
\text { (semanas) }\end{array}$} & \multicolumn{3}{|c|}{ Lote 1} & \multicolumn{3}{|c|}{ Lote 2} \\
\hline & \multicolumn{3}{|c|}{ Tempo de secagem (horas) } & \multicolumn{3}{|c|}{ Tempo de secagem (horas) } \\
\hline & 0 & 20 & 40 & 0 & 20 & 40 \\
\hline$\overline{0}$ & 45,9 & 39,4 & 36,1 & 45,2 & 39,3 & 35,1 \\
\hline 6 & 46,0 & 39,1 & 35,6 & 45,2 & 39,3 & 34,8 \\
\hline 12 & 46,1 & 39,1 & 35,3 & 46,5 & 38,9 & 35,6 \\
\hline 18 & 45,8 & 39,2 & 35,3 & 46,9 & 38,7 & 35,2 \\
\hline 24 & 45,3 & 40,4 & 36,3 & 46,1 & 41,0 & 36,4 \\
\hline 30 & 46,1 & 39,8 & 35,7 & 45,6 & 40,0 & 36,3 \\
\hline 36 & - & - & - & 45,9 & 39,7 & 36,3 \\
\hline 42 & - & - & - & 47,1 & 40,8 & 36,6 \\
\hline 48 & - & - & - & 46,3 & 41,2 & 36,2 \\
\hline 54 & - & - & - & 45,6 & 42,1 & 36,1 \\
\hline
\end{tabular}

Após a secagem, os três sublotes foram armazenados no escuro e à $15^{\circ} \mathrm{C}$, em sacos plásticos (20mm de espessura) fechados (Martins et al., 2000). As amostras foram retiradas periodicamente para os testes, independentemente de haver ou não sementes germinadas dentro da embalagem, assumindo-se que um armazenamento eficiente seria capaz de manter as sementes em condições de originar plântulas normais quando colocadas em substrato e ambiente próprio para a produção de mudas.

Durante o armazenamento foram realizadas as seguintes determinações em laboratório, a cada seis semanas, até o término das sementes: teor de água: avaliado pelo método da estufa a $105 \pm 3^{\circ} \mathrm{C} / 24$ horas (Brasil, 1992), utilizando-se quatro repetições de sete sementes inteiras; germinação: conduzido com quatro repetições de 20 sementes, mantidas nas temperaturas alternadas de 20-30C e luz (8h), em substrato vermiculita, esterilizada $\left(120^{\circ} \mathrm{C} / 12\right.$ horas), usando caixas plásticas transparentes $(11 \times 11 \times 3,5 \mathrm{~cm})$ (Brasil, 1992; Andrade et al., 1999). A contagem das plântulas foi realizada semanalmente, do sétimo ao $49^{\circ}$ dia após a semeadura, quando foram calculadas as porcentagens de germinação (plântulas normais); primeira contagem da germinação: realizada cinco dias após a semeadura, contando-se o número de botões germinativos emitidos; índice de velocidade de germinação (IVG): determinado por meio de adaptação do critério estabelecido por Maguire (1962), contando-se semanalmente as sementes germinadas após a instalação do teste de germinação.

A análise de variância foi realizada separadamente para cada teste, em delineamento inteiramente casualizado, com quatro repetições, em arranjo fatorial $3 \mathrm{x}$ 6 para L1 e 3 x 10 para L2, onde os fatores corresponderam a três tempos de secagem e a seis ou dez períodos de armazenamento, em semanas, que variaram em função da disponibilidade das sementes. A comparação entre as médias dos tratamentos foi efetuada por meio do teste de Tukey. Os dados em porcentagem foram transformados em arc sen (x $/ 100)^{1 / 2}$, previamente à análise.

\section{RESULTADOS E DISCUSSÃO}

A secagem inicial das sementes e a duração do armazenamento afetaram significativamente todas as características estudadas, exceto o teor de água, em ambos os lotes de sementes (Tabelas 1, 2 e 3).

A redução do teor de água das sementes, em relação ao teor inicial, foi de aproximadamente $13,6 \%$ e $21,8 \%$ para 20 e 40 horas de secagem, respectivamente (Tabela 1). O aumento do tempo de secagem resultou em sementes com teor de água decrescentes em ambos os lotes. Porém, o tempo de armazenamento praticamente não alterou o teor de água das sementes no lote 1 e no sublote de sementes não-secadas 
(0 horas) no lote 2 . Nos demais sublotes do lote 2, observou-se um leve aumento do teor de água (Tabela 1). $\mathrm{O}$ aumento do teor de água destas sementes pode estar relacionado a eventos metabólicos que ocorrem durante o processo de deterioração e ao aumento da incidência de microorganismos durante o armazenamento de sementes recalcitrantes, como também foi constatado em trabalhos com Ingá uruguensis Hook. Et Arn., Araucária angustifolia (Bert.) e Euterpe oleracea Mart. (Bilia et al., 1998; Piriz et al., 2003; Nascimento, 2006). Vale lembrar que, devido à disponibilidade, as sementes do lote 2 foram avaliadas por um período maior de armazenamento que as sementes do lote 1 (54 e 30 semanas, respectivamente), havendo maior tempo para que as sementes ficassem expostas ao microambiente criado dentro da embalagem plástica. De modo geral, as mé-
Tabela 2. Germinação em porcentagem de dois lotes de sementes de palmito-vermelho em função de períodos de secagem e armazenamento (Germination, in percentage, of two seed lots of Euterpe espiritosantensis as affected by drying period and storage). Botucatu, UNESP, $1998 / 2000$.

\begin{tabular}{|c|c|c|c|c|c|c|}
\hline \multirow{3}{*}{$\begin{array}{l}\text { Armazenamento } \\
\text { (semanas) }\end{array}$} & \multicolumn{3}{|c|}{ Lote 1} & \multicolumn{3}{|c|}{ Lote 2} \\
\hline & \multicolumn{3}{|c|}{ Tempo de secagem (horas) } & \multicolumn{3}{|c|}{ Tempo de secagem (horas) } \\
\hline & 0 & 20 & 40 & 0 & 20 & 40 \\
\hline 0 & $88,8 \mathrm{a} A$ & $85,0 \mathrm{a} A$ & $76,3 a \mathrm{~A}$ & $88,8 a b \mathrm{~A}$ & $92,5 a \mathrm{~A}$ & $88,8 \mathrm{a} A$ \\
\hline 6 & $83,8 a b \mathrm{~A}$ & $82,5 a \mathrm{~A}$ & $73,8 a b \mathrm{~A}$ & $98,8 a \mathrm{~A}$ & $87,5 a b A B$ & $81,3 a b$ B \\
\hline 12 & $83,8 \mathrm{ab} A$ & $80,0 a \mathrm{~A}$ & 71,3ab A & $91,3 a b$ A & $81,3 a b c A B$ & $75,0 b$ B \\
\hline 18 & $82,5 a b$ A & $76,3 a b$ A & $57,5 \mathrm{ab}$ B & $88,8 a b$ A & $82,5 a b c A B$ & $72,5 b$ B \\
\hline 24 & $82,5 a b$ A & $76,3 a b$ A & $53,8 a b$ B & $85,0 a b c$ A & $87,5 a b$ A & $20,0 \mathrm{c} \mathrm{B}$ \\
\hline 30 & $71,3 \mathrm{~b} A$ & $61,3 b \mathrm{AB}$ & $45,0 \mathrm{~b} B$ & $81,3 a b c$ A & $68,8 \mathrm{bcd} A$ & $10,0 \mathrm{~cd} \mathrm{C}$ \\
\hline 36 & - & - & - & $86,3 a b \mathrm{~A}$ & $61,3 \mathrm{~cd} \mathrm{~B}$ & $0,0 \mathrm{~d} \mathrm{~B}$ \\
\hline 42 & - & - & - & $70,0 \mathrm{bc} \mathrm{A}$ & $57,5 \mathrm{D} \mathrm{A}$ & $0,0 \mathrm{~d} \mathrm{~B}$ \\
\hline 48 & - & - & - & $61,3 \mathrm{~cd} \mathrm{~A}$ & $48,8 \mathrm{D} \mathrm{A}$ & $0,0 \mathrm{~d} \mathrm{~B}$ \\
\hline 54 & - & - & - & $43,8 \mathrm{~d} A$ & $48,8 \mathrm{D} \mathrm{A}$ & $0,0 \mathrm{~d} \mathrm{~B}$ \\
\hline CV\% & & 20,0 & & & 52,7 & \\
\hline
\end{tabular}

Médias seguidas de mesma letra minúscula na coluna e maiúscula na linha não diferem estatisticamente entre si, pelo teste Tukey, $\mathrm{p}<0,05$ (Means followed by the same small letter in the column and capital letter in the row did not differ from each other, Tukey's test, $\mathrm{p}<0.05)$. A análise foi realizada sobre dados transformados para arc sen $(\mathrm{x} / 100)^{1 / 2}$ (Analysis performed over data transformed to arc sen $\left.(\mathrm{x} / 100)^{1 / 2}\right)$.

Tabela 3. Vigor de sementes de palmito-vermelho avaliado pelos testes de primeira contagem (PC) e índice de velocidade de germinação (IVG) em dois lotes de sementes em função de períodos de secagem e armazenamento (Vigor of Euterpe espiritosantensis seeds evaluated by first counting of germination (PC) and germination rate (IVG) in two seed lots as affected by drying period and storage). Botucatu, UNESP, 1998/2000.

\begin{tabular}{|c|c|c|c|c|c|c|c|}
\hline \multirow{3}{*}{ Vigor } & \multirow{3}{*}{$\begin{array}{l}\text { Armazenamento } \\
\text { (semanas) }\end{array}$} & \multicolumn{3}{|c|}{ Lote 1} & \multicolumn{3}{|c|}{ Lote 2} \\
\hline & & \multicolumn{3}{|c|}{ Tempo de secagem (horas) } & \multicolumn{3}{|c|}{ Tempo de secagem (horas) } \\
\hline & & 0 & 20 & 40 & 0 & 20 & 40 \\
\hline & 0 & $53,8 a \mathrm{~A}$ & $8,8 \mathrm{a} B$ & $3,8 \mathrm{a} B$ & $67,5 \mathrm{Abc} A$ & $15,0 \mathrm{~b} B$ & $0,0 a \mathrm{~B}$ \\
\hline & 6 & $55,0 a \mathrm{~A}$ & $6,3 a b \mathrm{~B}$ & $3,8 \mathrm{a} B$ & $62,5 b c A$ & $31,3 a \mathrm{~B}$ & $0,0 a \mathrm{~B}$ \\
\hline & 12 & $50,0 a \mathrm{~A}$ & $3,7 a b B$ & 1,3a B & $68,3 \mathrm{Abc} A$ & 27,5 a B & $0,0 a \mathrm{~B}$ \\
\hline & 18 & $46,3 a \mathrm{~A}$ & $0,0 \mathrm{~b} B$ & $0,0 \mathrm{a} B$ & $73,8 a b \mathrm{~A}$ & $3,8 b c B$ & 0,0 a B \\
\hline PC & 24 & $45,0 \mathrm{a} A$ & $0,0 \mathrm{~b} B$ & 0,0 a B & $88,8 a \mathrm{~A}$ & $8,8 b c B$ & $0,0 \mathrm{a} B$ \\
\hline \multirow[t]{11}{*}{$(\%)$} & 30 & $43,8 a \mathrm{~A}$ & $0,0 \mathrm{~b} B$ & 0,0 a B & $78,8 a b \mathrm{~A}$ & $5,0 \mathrm{bc} \mathrm{B}$ & $0,0 \mathrm{a} B$ \\
\hline & 36 & - & - & - & $80,0 \mathrm{ab} A$ & $0,0 \mathrm{c} \mathrm{B}$ & $0,0 \mathrm{a} B$ \\
\hline & 42 & - & - & - & $72,5 a b A$ & $0,0 \mathrm{c} \mathrm{B}$ & $0,0 a$ B \\
\hline & 48 & - & - & - & $47,5 \mathrm{~cd} A$ & $0,0 \mathrm{c} \mathrm{B}$ & $0,0 a$ B \\
\hline & 54 & - & - & - & $40,0 \mathrm{~d} A$ & $0,0 \mathrm{c} \mathrm{B}$ & $0,0 a$ B \\
\hline & CV\% & & 130,0 & & & 133,3 & \\
\hline & 0 & $18,0 \mathrm{a} A$ & $11,4 \mathrm{ab} B$ & $6,7 \mathrm{~b} C$ & $10,1 \mathrm{de} A$ & $9,0 \mathrm{a} A B$ & $7,9 a$ B \\
\hline & 6 & $17,3 a b \mathrm{~A}$ & $13,3 a$ B & $9,6 a \mathrm{C}$ & $16,1 \mathrm{ab} A$ & $8,5 a$ B & $6,5 b \mathrm{C}$ \\
\hline & 12 & $16,7 \mathrm{ab} A$ & $10,2 \mathrm{ab} B$ & $5,8 \mathrm{bc} \mathrm{C}$ & $15,8 \mathrm{ab} A$ & 8,1 a B & $5,8 \mathrm{bc} \mathrm{C}$ \\
\hline & 18 & $15,9 a b A$ & $10,9 a b$ B & $5,3 \mathrm{bcd} C$ & $16,7 a b A$ & $7,8 a b$ B & $5,2 \mathrm{c} \mathrm{C}$ \\
\hline & 24 & $15,2 \mathrm{bc} \mathrm{A}$ & $9,1 b$ B & $4,2 \mathrm{~cd} \mathrm{C}$ & $17,8 \mathrm{a} A$ & 9,0 a B & $2,0 \mathrm{~d} C$ \\
\hline \multirow[t]{6}{*}{ IVG } & 30 & $13,0 \mathrm{c} \mathrm{A}$ & $8,3 b$ B & $3,4 d \mathrm{C}$ & $16,3 a b$ A & $7,4 a b$ B & $1,2 \mathrm{~d} C$ \\
\hline & 36 & - & - & - & $13,8 b c A$ & $4,2 \mathrm{c} \mathrm{B}$ & 0,2 e C \\
\hline & 42 & - & - & - & $12,2 \mathrm{~cd} \mathrm{~A}$ & $5,6 b c B$ & 0,0 e C \\
\hline & 48 & - & - & - & 9,2 de $A$ & 7,0ab A & 0,0 e B \\
\hline & 54 & - & - & - & $6,8 \mathrm{e} A$ & $5,7 \mathrm{bc} \mathrm{A}$ & 0,0 e B \\
\hline & CV\% & & 43,3 & & & 67,0 & \\
\hline
\end{tabular}

Médias seguidas de mesma letra minúscula na coluna e maiúscula na linha não diferem estatisticamente entre si, pelo teste Tukey, p $<0,05$ (Means followed by the same small letter in the column and capital letter in the row did not differ from each other, Tukey's test, $\mathrm{p}<0.05$ ). A análise foi realizada sobre dados transformados para arc sen $(\mathrm{x} / 100)^{1 / 2}$ (Analysis performed over data transformed to arc sen $\left.(\mathrm{x} / 100)^{1 / 2}\right)$. 
dias de teor de água para ambos lotes foram similares, com valores de 46; 40 e $36 \%$, respectivamente, para $0 ; 20$ e 40 horas de secagem (Tabela 1).

Para sementes não-armazenadas (0 semanas de armazenamento), verificouse que a secagem reduziu de modo significativo o vigor mas não comprometeu a germinação (Tabelas 2 e 3). Para as sementes do Lote L1, quando o vigor foi avaliado através do Índice de Velocidade de Germinação (IVG), a redução foi tanto maior, quanto maior o período de dessecação, o que ratifica o caráter recalcitrante de sementes dessa espécie (Martins et al., 1999a). Em sementes recalcitrantes a água subcelular está fortemente associada às superfícies macromoleculares assegurando, em parte, a estabilidade de membranas e macromoléculas. A perda de água estrutural durante o processo de secagem de sementes recalcitrantes causaria a alteração de sistemas metabólicos e das membranas celulares, resultando no início do processo de deterioração (Farrant et al., 1988). Esse processo resulta inicialmente na redução de vários atributos de desempenho e vigor da semente e, finalmente, na perda da capacidade germinativa, como também verificado em outros trabalhos envolvendo a secagem de sementes de Euterpe spp (Andrade, 1994, Martins et al., 1999 acb, 2000; Nascimento, 2006).

Para ambos os lotes estudados foi constatado efeito do armazenamento sobre a germinação e sobre o índice de velocidade de germinação, para sementes submetidas a todos os tempos de secagem (Tabelas 2 e 3), tendo-se observado redução na qualidade das sementes com o decorrer do tempo de armazenamento. No entanto, para L1, só houve diferença significativa em germinação em relação às sementes não armazenadas para 30 semanas de armazenamento, para todos os tempos de secagem (Tabela 2). Para a primeira contagem de germinação só foi observado efeito do armazenamento para sementes submetidas a 20 horas de secagem (Tabela 3). As sementes que não foram submetidas à secagem mantiveram os resultados de primeira contagem ao longo do período de armazenamento. Já as sementes submetidas a 40 horas de secagem apresentaram resultados muito baixos para a primeira contagem logo na primeira avaliação. Desta forma, a redução que porventura pudesse ser causada pelo armazenamento não se manifestou.

Para o lote 2, observou-se efeito mais intenso da secagem sobre a qualidade das sementes armazenadas. A germinação das sementes submetidas à secagem por $0 ; 20$ e 40 horas diferiu significativamente da germinação das sementes que não passaram por armazenamento a partir de 42; 30 e 12 semanas, respectivamente, sendo sempre menor à medida em que o tempo de armazenamento aumentou (Tabela 2). Para a primeira contagem de germinação, as sementes armazenadas diferiram significativamente daquelas que não passaram por armazenamento com $54 \mathrm{e}$ a partir de 30 semanas de armazenamento para sementes submetidas à secagem por 0 e 20 horas, respectivamente. Nas sementes submetidas a 40 horas de secagem não foram observadas plântulas na data da primeira contagem, independente do período de armazenamento das sementes. Já o Índice de Velocidade de Germinação (IVG) apresentou redução de forma mais drástica quanto menor foi o teor inicial de água nas sementes. Assim, pode-se afirmar que a germinação e o vigor de lotes de sementes de $E$. espiritosantensis dependem do teor de água inicial e do tempo de armazenamento.

A longevidade dos lotes e sublotes avaliados foi distinta (Tabela 2). Para o lote 1 observou-se a presença de sementes com capacidade de germinar após os tempos de secagem até 30 semanas de armazenamento, período em que o estoque de sementes terminou (germinação de $71 ; 61$ e $45 \%$, respectivamente, para 0; 20 e 40 horas). O lote 2 apresentou perda total da viabilidade após a $30^{\text {a }}$ semana somente para as sementes do sublote submetido a 40 horas de secagem (teor de água de $36 \%$ ), enquanto as sementes dos demais sublotes mantiveram-se viáveis até 54 semanas de armazenamento (germinação igual a 46\%).

Quanto ao efeito da secagem sobre as sementes não armazenadas, Martins et al. (1999a e 2000) verificaram que sementes de E. espiritosantensis apresentam alta porcentagem de germinação
(90,0\% a $87,5 \%)$ quando não desidratadas (teor de água de 46,6\% a 51,4\%). Por outro lado, as sementes têm a viabilidade reduzida quando o teor de água atinge valores abaixo desta faixa. No entanto, no presente trabalho, as sementes de ambos os lotes, desidratadas até 35 e $36 \%$ de água, não apresentaram danos de secagem avaliados pelo teste de germinação, mas sim efeitos latentes, com o aumento do tempo de armazenamento, mesmo em sementes que mantiveram-se com teores de água relativamente altos, de 45 a $46 \%$. A existência de uma faixa, e não de um valor único, para teores crítico e letal de água, pode ser atribuída às sensibilidades diferenciadas na tolerância à perda de água observadas dentro de uma mesma espécie, de acordo com o habitat de origem e à diversidade genética. Este comportamento já havia sido verificado para sementes recalcitrantes de $E$. espiritosantensis (Martins et al.,1999a) e de outras espécies de palmeiras, como Elaeis guinensis (Ellis et. al., 1991), Euterpe edulis e E. oleracea (Martins et al.,1999bc) e Bactris gasipae Kunth. (Bovi et al., 2004). Esses fatores podem explicar, em parte, também, o comportamento diverso entre as sementes dos lotes L1 e L2 no presente estudo, em função da secagem. Outro fator que merece maiores estudos é o efeito da variação climática. Vale ressaltar que as plantas matrizes colhidas produziram sementes em épocas distintas: o lote L1 foi obtido na época das águas e, L2, na estação seca.

Com base nos resultados obtidos pode-se concluir que sementes de palmiteiro-vermelho devem ser armazenadas sem secagem, com $45-46 \%$ de teor de água.

\section{AGRADECIMENTOS}

Agradecemos pelo financiamento desta pesquisa através do projeto jovem pesquisador da FAPESP.

\section{REFERÊNCIAS}

ANDRADE ACS. 1994. Efeito da secagem e do armazenamento sobre a germinação, o vigor de plântulas e a integridade do sistema de membranas em sementes de palmito (Euterpe edulis Mart.). Rio de Janeiro: Universidade Federal do Rio de Janeiro. 87p. (Dissertação mestrado) 
ANDRADE ACS; LOUREIRO MB; SOUZA ADO; RAMOS FN; CRUZ APM. 1999. Reavaliação do efeito do substrato e da temperatura na germinação de sementes de palmiteiro (Euterpe edulis M.). Revista Árvore, 23: 279-283.

BARBEDO CJ; MARCOS FILHO J. 1998. Tolerância à dessecação em sementes. Acta Botânica Brasileira, 12: 145-164.

BILIA DAC. 1997. Tolerância à dessecação $e$ armazenamento de sementes de Inga uruguensis Hook Et Arn. Piracicaba: USPESALQ. 88p (Dissertação mestrado).

BILIA DAC; MARCOS FILHO J; NOVEMBRE ADLC. 1998. Conservação da qualidade fisiológica de sementes de Inga uruguensis Hook Et Arn. Revista Brasileira de Sementes, 20: 4854.

BOVI MLA; GODOY-JÚNIOR G; SAES LA. 1987. Pesquisas com os gêneros Euterpe e Bactris no Instituto Agronômico de Campinas. Agronômico, 39: 129-174.

BOVI MLA; VAL MR; DIAS GS; SPIERING SH. 1994. Floral biology and reproductive system of Euterpe espiritosantensis Fernandes. Acta Horticulturae, 360: 41-56.

BOVI MLA; MARTINS CC; SPIERING SH 2004. Desidratação de sementes de quatro lotes de pupunheira: efeitos sobre a germinação e o vigor. Horticultura Brasileira, 22: 109-112.

BRASIL. Ministério da Agricultura e Reforma Agrária. 1992. Regras para análise de sementes. Brasília: SNDA/DNDV/CLAV. 365p.
CHIN HF. 1994. Storage of recalcitrant seeds. In: Seed quality: basic mechanisms and agricultural implications. New York: Food Products Press. p. 209-222.

ELLIS RH; HONG TD; ROBERTS EH SOETISNA U. 1991. Seed storage behaviour in Elaeis guineensis. Seed Science Research, 1: 99-104.

FARRANT JM; PAMMENTER NW; BERJAK P. 1988. Recalcitrance - a current assessment. Seed Science and Technology, 16: 155-166.

FERNANDES HQB. 1989. Uma nova espécie de Euterpe (Palmae - Arecoideae - Areceae) do Brasil. Acta Botânica Brasilica, 3: 43-49.

GENTIL DFO. 2003. Conservação de sementes de Myrciaria dubia (H.B.K.) McVAUGH. Piracicaba: USP-ESALQ. 42p. (Tese mestrado)

HOR YL; CHIN HF; KARIN MZ. 1984. The effect of seed moisture and storage temperature on the storability of cocoa (Theoboma cacao) seeds. Seed Science and Technology, 12: 415420.

MAGUIRE JD. 1962. Speed of germination aid in selection and evaluation for emergence and vigour. Crop Science, 2: 176-177.

MARTINS CC; NAKAGAWA J; BOVI MLA. 1999a. Tolerância à dessecação de sementes de palmito-vermelho (Euterpe espiritosantensis Fernandes). Revista Brasileira de Botânica, 22: 391-396.
MARTINS CC; NAKAGAWA J; BOVI MLA. 1999b. Dessication tolerance of four seeds lots of Euterpe edulis Mart. Seed Science and Technology, 28: 1-13, 1999b.

MARTINS CC; BOVI MLA; NAKAGAWA J; GODOY JRG. 2000. Despolpamento e temperatura no armazenamento temporário de sementes de palmito-vermelho (Euterpe espiritosantensis Fernandes). Revista Brasileira de Sementes, 22: 169-176.

MARTINS CC; NAKAGAWA J; BOVI MLA; STANGUERLIM H. 1999c. Teores de água crítico e letal para sementes de açaí (Euterpe oleracea Mart. - Palmae). Revista Brasileira de Sementes, 21: 125-132.

NASCIMENTO WMO. 2006. Conservação de sementes de açaí (Euterpe oleracea Mart.). Piracicaba: USP-ESALQ. 60f (Tese mestrado)

NODARI RO; FANTINI AC; GUERRA MP; REIS MS; SCHUCH O. 1998. Conservação de frutos e sementes de palmiteiro (Euterpe edulis Mart.) sob diferentes condições de armazenamento. Revista Árvore, 22: 1-10.

PIRIZ CARILLO V; CHAVES A; FASSOLA H; MUGRIDGE A. 2003. A refrigerated storage of seeds of Araucaria angustifolia (Bert.) O. Kuntze over a period of 24 months. Seed Science and Technology, 31: 411-429. 